\title{
MicroRNA responses to focal cerebral ischemia in male and female mouse brain
}

\author{
Theresa A. Lusardi ${ }^{1+}$, Stephanie J. Murphy ${ }^{2+}$, Jay I. Phillips ${ }^{2}$, Yingxin Chen ${ }^{2}$, Catherine M. Davis ${ }^{2}$, \\ Jennifer M. Young ${ }^{2}$, Simon J. Thompson ${ }^{1}$ and Julie A. Saugstad ${ }^{2 *}$ \\ Dow Neurobiology Laboratories, Legacy Research Institute, Portland, OR, USA \\ ${ }^{2}$ Department of Anesthesiology and Perioperative Medicine, Oregon Health and Science University, Portland, OR, USA
}

\section{Edited by:}

Hermona Soreq, The Hebrew

University of Jerusalem, Israel

Reviewed by:

Enrico Tongiorgi, University of

Trieste, Italy

Alessandro Vercelli, University of Torino, Italy

*Correspondence:

Julie A. Saugstad, Department of Anesthesiology and Perioperative

Medicine, Oregon Health and

Science University, 3181 SW Sam

Jackson Park Road, HRC5N,

Portland, OR 97239-3098, USA

e-mail:saugstad@ohsu.edu

${ }^{t}$ These authors have contributed

equally to this work.
Stroke occurs with greater frequency in men than in women across diverse ethnic backgrounds and nationalities. Work from our lab and others have revealed a sex-specific sensitivity to cerebral ischemia whereby males exhibit a larger extent of brain damage resulting from an ischemic event compared to females. Previous studies revealed that microRNA (miRNA) expression is regulated by cerebral ischemia in males; however, no studies to date have examined the effect of ischemia on miRNA responses in females. Thus, we examined miRNA responses in male and female brain in response to cerebral ischemia using miRNA arrays. These studies revealed that in male and female brains, ischemia leads to both a universal miRNA response as well as a sexually distinct response to challenge. Target prediction analysis of the miRNAs increased in male or female ischemic brain reveal sex-specific differences in gene targets and protein pathways. These data support that the mechanisms underlying sexually dimorphic responses to cerebral ischemia includes distinct changes in miRNAs in male and female brain, in addition to a miRNA signature response to ischemia that is common to both.

Keywords: microRNA, cerebral ischemia, sex-differences, array analysis, qRT-PCR, pathway analysis, stroke

\section{INTRODUCTION}

Stroke occurs more frequently in men than women across diverse ethnic backgrounds and nationalities (Bushnell, 2008; Reeves et al., 2008; Saini and Shuaib, 2008; Appelros et al., 2009; Persky et al., 2010; Ovbiagele et al., 2013; Towfighi et al., 2013). Our lab and others have shown that sensitivity to cerebral ischemia, i.e., the extent of brain damage resulting from ischemic insult, is sex-specific, with female animals being less sensitive than males (Murphy et al., 2004; Koerner et al., 2007; Lang and McCullough, 2008; Reeves et al., 2008; Vagnerova et al., 2008; Cheng and Hurn, 2010; Siegel et al., 2010). Sex-specific responses are also observed in response to focal cerebral ischemia in isoflurane preconditioned mice (Kitano et al., 2007) and in immune responses to ischemia (Banerjee et al., 2013). Furthermore, sex differences in ischemic sensitivity have been extended to the cellular level as our lab and others have shown that astrocytes (Liu et al., 2007, 2008) and neurons (Li et al., 2005; Johnsen and Murphy, 2011) from male newborn rodents are more sensitive to oxygen-glucose deprivation (an in vitro model of ischemia) than cells from female newborn rodents. These observations suggest that the male brain exhibits a more "ischemia-sensitive" phenotype than the female brain. However, the underlying molecular mechanisms for this sexually dimorphic response to ischemia are not well understood.

We examined a role for miRNAs in ischemic responses in the male and female brain. MiRNAs are short, non-coding RNA sequences that regulate post-transcriptional gene expression via translational repression or mRNA degradation (Ambros, 2004; Murchison and Hannon, 2004; Niwa and Slack, 2007; Guarnieri and DiLeone, 2008; Chua et al., 2009). MiRNAs have been implicated in the regulation of numerous physiological and pathological processes such as brain differentiation (Feng and Feng, 2011), neurological disorders (Saugstad, 2010), ischemic preconditioning (Lusardi et al., 2010), and stroke (Rink and Khanna, 2011; Tan et al., 2011). The few studies which have examined miRNA responses to injury in brain have either focused on irradiation injury (Ilnytskyy et al., 2008; Koturbash et al., 2011), evaluated a single miRNA target of interest following brain ischemia (Siegel et al., 2011), or profiled miRNAs in male ischemic brain without linking them functionally to ischemic mechanisms and outcomes (Jeyaseelan et al., 2008; Dharap et al., 2009; Liu et al., 2010; Lusardi et al., 2010).

For these studies we focused on miRNA expression at $8 \mathrm{~h}$ after ischemia, based on our previous miRNA studies in rodent brain showing that this reperfusion time is optimal for robust change in miRNA expression levels. Two previous studies revealed little or no changes in miRNA expression at 2 and $4 \mathrm{~h}$ after treatment, robust changes $8 \mathrm{~h}$ after treatment, and a return to levels comparable to naïve controls by $24 \mathrm{~h}$ after treatment (Lusardi et al., 2010, 2012). These studies suggest that the treatments used (ischemia or glutamate activation) induced transcriptional changes in miRNA expression, or alterations in the miRNA processing pathway, that were optimally detected $8 \mathrm{~h}$ after the treatment. This time course would be consistent with miRNAs as early mediators of mRNA translation and protein expression that in turn lead to cellular changes that develop within 24-72 $\mathrm{h}$ after ischemia.

Our miRNA profiling studies revealed that there are sexspecific differences in miRNA responses to ischemia as well as a universal, ischemia-induced miRNA signature equally present in 
both male and female brains. Our findings reveal a novel mechanism, namely the differential regulation of miRNA responses, for sex differences in ischemic sensitivity mediated by sex-specific miRNA pathways in male and female brain.

\section{MATERIALS AND METHODS EXPERIMENTAL GROUPS}

Experiments were carried out in male and female C57BL/6 mice (Charles River Laboratories, Wilmington, MA, USA), 8-14 weeks of age and weighing 20-25 g. Experiments were carried out in accordance with the National Institutes of Health guidelines for research animal care and approved by the Oregon Health and Science University Animal Care and Use Committee. All mice were maintained on a 12/12 h light-dark cycles and permitted ad libitum access to food and water. Male and female mice were randomized to one of the following experimental groups: control (experimentally naïve), sham surgery, or transient focal cerebral ischemia.

\section{TRANSIENT FOCAL CEREBRAL ISCHEMIA}

All surgeries were conducted under aseptic conditions by a single surgeon. Transient focal cerebral ischemia was induced in male and female mice for $60 \mathrm{~min}$ by reversible right middle cerebral artery occlusion (MCAO) under isoflurane anesthesia, followed by $8 \mathrm{~h}$ of reperfusion as previously described (Chen et al., 2012). Peri-ischemic head and body temperature were controlled at 36.5 $\pm 1.0^{\circ} \mathrm{C}$ (mean \pm standard deviation) with warm water pads and a heating lamp. The common carotid artery was temporarily occluded while a 6-0 nylon monofilament surgical suture (ETHICON, Inc., Somerville, NJ, USA) with a silicone-coated (Xantopren Comfort Light, Heraeus Kulzer, Germany) tip was inserted via an external carotid artery stump distal to the internal carotid artery to the origin of the middle cerebral artery. After $60 \mathrm{~min}$ of MCAO, the filament was withdrawn to allow for reperfusion. All incisions were the closed with 6-0 surgical sutures (ETHICON, Inc., Somerville, NJ, USA) before each mouse was awakened and recovered in a separate cage with a warm water pad. For sham surgeries, the filament was placed but not advanced to achieve MCAO. Occlusion and reperfusion were verified in each mouse by laser Doppler flowmetry (LDF) (Model DRT4, Moor Instruments Inc. Wilmington, USA). Mice were excluded if intra-ischemic LDF (\% pre-ischemic LDF baseline) was greater than $25 \%$. Neurological deficit scores were determined at $1 \mathrm{~h}$ of reperfusion to confirm the presence of ischemic injury using a $0-4$ point scale as follows: 0 , no neurological dysfunction; 1 , failure to extend left forelimb fully when lifted by tail; 2 , circling to the contralateral side; 3 , falling to the left; and 4, no spontaneous movement or in a comatose state (Chen et al., 2012). Any animal without a deficit at $1 \mathrm{~h}$ of reperfusion was excluded from the study. Eight hours following either sham surgery or focal cerebral ischemia, mice were anesthetized with isoflurane and euthanized by decapitation. Experimentally naïve mice were also anesthetized with isoflurane and euthanized by decapitation. Right and left cortices were sub-dissected from each mouse brain, and the tissues were frozen in 2 methyl-2-butane on dry ice, then stored at $-80^{\circ} \mathrm{C}$.

\section{RNA ISOLATION}

To correlate with the right MCAO model, RNA was isolated from the right mouse brain cortex with the mirVana miRNA Isolation Kit (Life Technologies, Carlsbad, CA, USA), following the recommended protocol for total RNA isolation from frozen tissue. The RNA isolation did not include the "Enrichment Procedure for Small RNA" in the protocol provided with the kit. Total RNA was eluted with $100 \mu \mathrm{L}$ of Elution Solution provided with the RNA isolation kit, and the RNA concentrations quantified by spectroscopic measurement of A260. RNA samples were stored at $-80^{\circ} \mathrm{C}$ until further use.

\section{MICRORNA ARRAY PROFILING}

Mouse MicroRNA Genome V2.0 PCR Arrays (MAM-200C; SABiosciences/Qiagen, Valencia, CA) were used to quantitatively assay miRNA expression in mouse brain. The arrays consisted of the 528 most abundantly expressed and well-characterized miRNA sequences in the mouse genome, as annotated by the Sanger miRBase Release 14. For qRT-PCR array analysis we used total RNA samples representing control male and female mice, and ischemic male and female mice. The RT2 miRNA First Strand Kit (SABiosciences) was used for Reverse Transcription (RT) of the RNAs, as per the manufacturer's instructions. Total RNA from control and ischemic male and female mice ( $n=3$ mice/group) were pooled, then $2 \mu \mathrm{g}$ of the pooled total RNA was incubated in RT buffers at $37^{\circ} \mathrm{C}$ for $2 \mathrm{~h}$, followed by $95^{\circ} \mathrm{C}$ for $5 \mathrm{~min}$ to degrade the RNA and to inactivate the reverse transcriptase. The first-strand cDNA samples were chilled on ice then diluted with RNase-free water. The RT2 SYBR green master mix (PA-012) was used for the qRT-PCR reactions, as per the manufacturer's instructions (SABiosciences). Briefly, the diluted first-strand cDNA was combined with master mix, then $25 \mu \mathrm{L}$ was aliquoted into each well of six 96 well plates. The six plates were briefly centrifuged then stored at $-20^{\circ} \mathrm{C}$. For amplification, individual plates were removed from $-20^{\circ} \mathrm{C}$, defrosted for $5 \mathrm{~min}$ at $\mathrm{RT}$, briefly centrifuged and placed into a 7500 Fast Real-Time PCR System (Applied Biosystems, Foster City, CA). Parameters were set to: (i) one cycle at $95^{\circ} \mathrm{C}$ for $10 \mathrm{~min}$, (ii) 40 cycles at $95^{\circ} \mathrm{C}$ for $15 \mathrm{~s}, 60^{\circ} \mathrm{C}$ for $40 \mathrm{~s}$, and $72^{\circ} \mathrm{C}$ for $30 \mathrm{~s}$. A dissociation step set to: (iii) $95^{\circ} \mathrm{C}$ for $15 \mathrm{~s} ; 60^{\circ} \mathrm{C}$ for $1 \mathrm{~min}$ and $95^{\circ} \mathrm{C}$ for $15 \mathrm{~s}$ was performed to ensure that all PCRs generated a single product. Normalized delta CT $(\Delta \mathrm{Ct})$ values were calculated with respect to the average of all $\mathrm{Ct}$ values, and $\Delta \Delta \mathrm{Ct}$ was calculated as $(\Delta \mathrm{Ct}$ Ischemic minus $\Delta$ Ct-Control) for all of the male and female miRNAs.

\section{SABIOSCIENCES qRT-PCR MICRORNA ARRAY NORMALIZATION}

We calculated the $\triangle \mathrm{Ct}$ for each miRNA in a given experiment based on the average Ct value of all miRNAs in that experimental condition: $\Delta$ Ctmir $=$ Ctavg - Ctmir. We then determined the response to ischemia for males and females separately, and calculated the change in miRNA expression as $\Delta \Delta$ Ctmir $=$ $\Delta$ Ctmirischemia $-\Delta$ Ctmircontrol. We defined statistical significance as a multiple of a standard deviation from the mean $\Delta \Delta \mathrm{Ct}(\mathrm{SD})$. 


\section{REAL-TIME qRT-PCR DETECTION}

We analyzed miRNA expression in individual mice (each group) from control $(n=5)$, sham $(n=5)$, and ischemic $(n=4)$ cortices. Sham surgery groups were added after the initial miRNA array profiling to account for any effects due to anesthesia and surgical stress. Detection of miRNAs was completed with a 2step qRT-PCR assay, using the miScript PCR System (Qiagen, Valencia, CA). One microgram of total RNA was converted to cDNA, for RNA from each individual sample, with the miScript II RT Kit, following the standard protocol with the miScript HiSpec Buffer. Negative control samples included those with no reverse transcriptase (No RT). The qRT-PCR assays were prepared for each individual using $1 \mu \mathrm{g}$ of total RNA in the same reverse transcription reaction protocol, omitting the RT enzyme in the reaction mix. The resulting $20 \mu \mathrm{L}$ of RT products (or No RT controls) were diluted to a total volume of $100 \mu \mathrm{L}$ with the addition of $80 \mu \mathrm{L}$ RNase/ DNase Free water, and stored at $-80^{\circ} \mathrm{C}$ before use in PCR assays. Two micro litres of cDNA was assayed in each PCR reaction well. miScript PCR Primer Assays (Qiagen) were used for detection of mature miRNA, using the miScript SYBR Green PCR Kit for qRT-PCR assays. The following primer sets were used:

$\begin{array}{ll}\text { miR-15b } & \text { 5' CGAAUCAUUAUUUGCUGCUCUA } \\ \text { miR-125b-3p } & \text { 5' ACGGGUUAGGCUCUUGGGAGCU } \\ \text { miR-296-5p } & \text { 5' AGGGCCCCCCCUCAAUCCUGU } \\ \text { miR-509-3p } & \text { 5' UGAUUGACAUUUCUGUAAUGG } \\ \text { miR-682 } & \text { 5' CUGCAGUCACAGUGAAGUCUG } \\ \text { miR-686 } & \text { 5' AUUGCUUCCCAGACGGUGAAGA } \\ \text { miR-883a-3p } & \text { 5' UAACUGCAACAGCUCUCAGUAU } \\ \text { miR-883b-3p } & \text { 5' UAACUGCAACAUCUCUCAGUAU } \\ \text { miR-1224 } & \text { 5' GUGAGGACUGGGGAGGUGGA }\end{array}$

(\#MS00011242) (\#MS00024066) (\#MS00016436) (\#MS00012306) (\#MS00033019) (\#MS00002821) (\#MS00012845) (\#MS00012859) (\#MS00011074)

Assays were performed with the standard recommended reaction mix $(25 \mu \mathrm{L}$ volume per reaction) in 96-well reaction plates, using a ViiA 7 Real Time PCR Detection System (Life Technologies). Each miRNA assay was performed in triplicate for individual samples. The $\mathrm{Ct}$ values were calculated using the same cycle threshold and baseline for all reactions. The $\Delta$ Ctmir $=$ Ctnon-changers - Ctmir, responses to ischemia for males and females were calculated the change in miRNA expression as $\Delta \Delta$ Ctmir $=\Delta$ Ctmirischemia $-\Delta$ Ctmircontrol, and $\Delta$ Ctmirischemia $-\Delta$ Ctmirsham.

\section{MICRORNA TARGET PREDICTION}

We used the miRmap target prediction program (http://mirmap. ezlab.org/, accessed November 2013) to query the predicted targets of miRNAs increased by ischemia in male and female brain (Vejnar et al., 2013). We then used the predicted protein targets identified in miRmap to query the PANTHER v8.1 program (http://www.pantherdb.org/, accessed November 2013). The PANTHER (Protein ANalysis THrough Evolutionary Relationships) classification system was designed to classify proteins (and their genes) according to: family and subfamily, molecular function, biological process, and pathway that explicitly specifies the relationships between the interacting molecules (Mi and Thomas, 2009; Mi et al., 2013).

\section{RESULTS}

\section{STATISTICAL ANALYSIS OF MICRORNA ARRAYS}

We used the Mouse MicroRNA Genome V2.0 PCR Arrays to profile the expression of mouse cortical miRNAs in both male and female brains. For the array studies, we pooled RNA samples isolated from control and ischemic male and female cortices. For array normalization, we first considered the endogenous controls included on the PCR arrays (Snord85, Snord68, Snord66, and Rnu6). We found that within a given treatment group, the endogenous controls were similar from plate-to-plate. However, across experimental groups the response of the endogenous controls was not consistent for sex or stroke groupings. We therefore calculated the $\Delta \mathrm{Ct}$ for each miRNA in a given experiment (control or ischemia) based on the average Ct value of all miRNAs in that experimental condition: $\Delta \mathrm{CtmiR}=\mathrm{Ctavg}-\mathrm{CtmiR}$, consistent with the assumption that most miRNAs would not be altered by focal ischemia as shown by linear regression analysis in Figure 1.

We then determined the response to ischemia for males and females separately, and calculated the change in miRNA expression as $\Delta \Delta$ CtmiR $=\Delta$ CtmiRischemia $-\Delta$ CtmiRcontrol. We defined significance as a multiple of a standard deviation from the mean $\Delta \Delta \mathrm{Ct}(\mathrm{SD})$, as shown in Figure 2. A miRNA was considered to be significantly decreased in response to ischemia if $\Delta \Delta$ Ctmir $(\leq-1.5 S D)$; the specific miRNAs are listed in Table 1A. A miRNA was considered to be significantly increased in response to ischemia if $\Delta \Delta$ Ctmir $(\geq 1.5 S D)$; specific miRNAs are listed in Table 1B. Approximately half of the $528 \mathrm{miRNAs}$ did not change in response to ischemia based on the cutoff criterion $(-0.5 S D)<\Delta \Delta$ Ctmir $<(0.5 S D)$. An additional group of miRNAs possibly changed in response to ischemia, with a (0.5 SD) $<\mid \Delta \Delta$ Ctmir $\mid<(1.5 \times S D)$, but did not meet the criterion for significance and were excluded from further analysis in this study.

For initial studies, miRNA was considered significantly changed if $\Delta \Delta \mathrm{Ct}$ was greater than $1.5 \mathrm{SD}$ (standard deviation) from the mean $\Delta \Delta \mathrm{Ct}$. The studies revealed two profiles: (1) sexdependent responses wherein ischemia-regulated miRNAs were unique to male or female brain, and (2) a sex-independent response in which miRNAs are equally present and equally regulated in both male and female brains (the focus of another study). Of the significantly increased miRNAs, $>50 \%$ were present in both male and female brain (Table 1 ).

\section{INDIVIDUAL MICRORNA VALIDATION}

Individual miRNAs representing down- and up-regulated species in male and female mice were further validated by Taqman qRTPCR assays (Qiagen). Although the initial miRNA array studies did not include a sham surgery group, we did include sham surgery groups in the individual qRT-PCR validation studies to account for any treatment effects due to anesthesia and surgical stress. As indicated in Table 1, we examined miR-883b-3p that decreased in males and increased in females, miR-296-5p that decreased in males and females, miR-509-3p that decreased in females (1A). We also examined miR-682 that increased in males, miR-686 and miR-1224 that increased in males and females, and miR-883a-3p that increased in females (1B). Bold font in Table 1 highlights the miRNAs that were chosen for individual qRT-PCR 

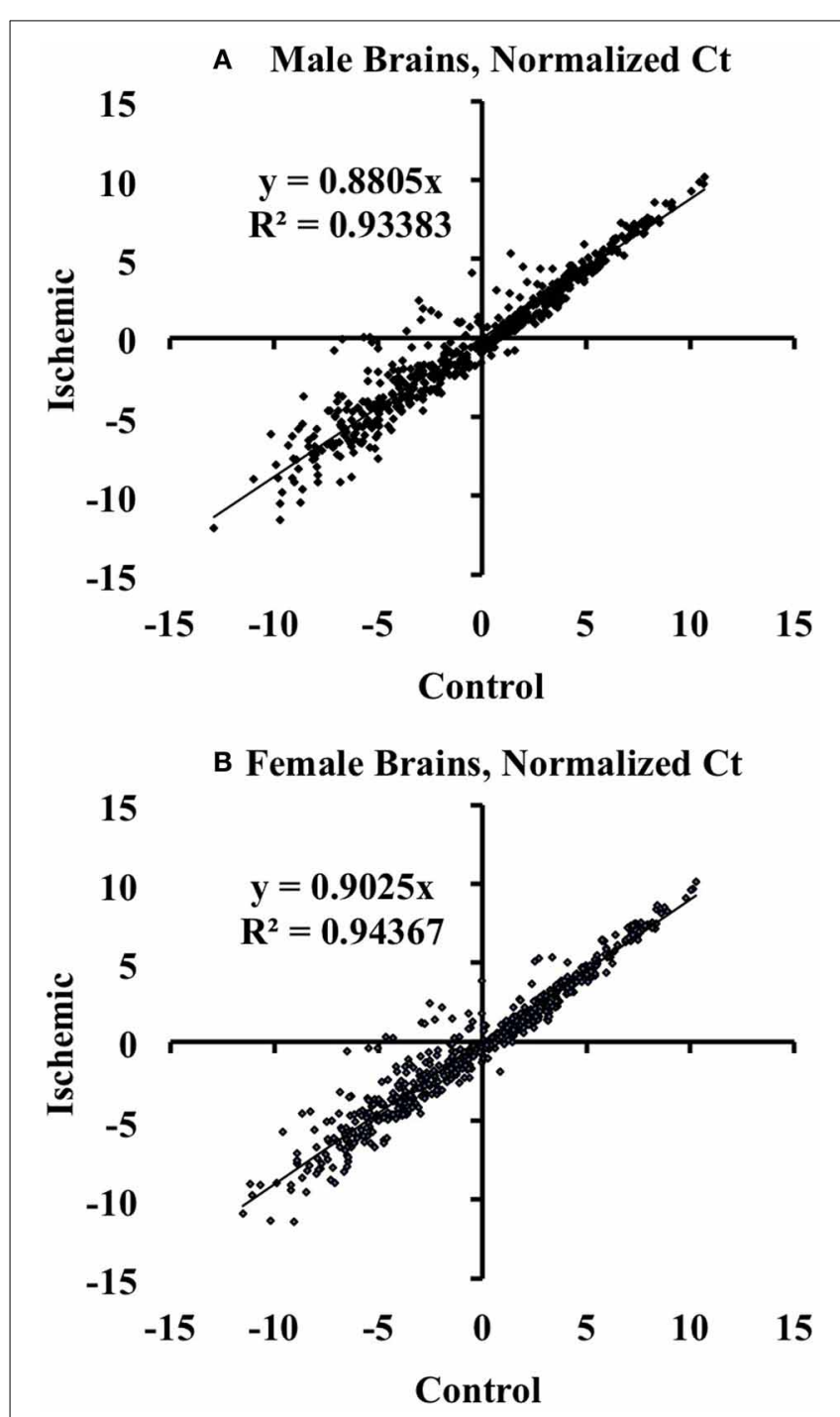

FIGURE 1 | Linear regression analysis of miRNAs altered by ischemia vs. control mouse cortices. The graphs illustrate that there are changes in miRNA expression in ischemia relative to control in male (A) and female (B) mouse cortex. The graphs also show that the majority of the assayed miRNAs are not altered by ischemia.

validation, while italic font indicates the miRNAs that showed significant changes in the opposite direction in male and female brain.

We calculated the $\Delta \Delta$ Cts using both control ( $n=5$ each male and female) and sham surgery ( $n=5$ each male and female) $\Delta \mathrm{Ct}$ values, relative to ischemia ( $n=4$ each male and female). Based on the array results, we selected miR-15b* and miR-125b-3p as candidates for a normalizing factor for our qRT-PCR validation. The rationale for this choice is as follows. We compared the raw $\mathrm{Ct}$ values for each of these miR across experimental conditions (control, sham, and ischemic) using a 2-Way repeated measures ANOVA (Prism, GraphPad Software, Inc. La Jolla, CA). The results showed: (1) there is no effect of the experimental condition (i.e., ischemia vs. sham vs. control) $(p=0.1429)$, there is

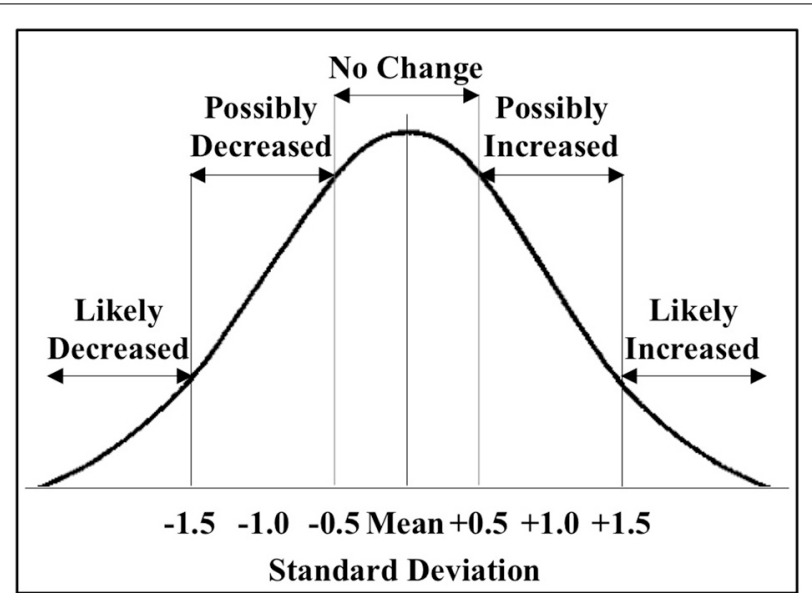

FIGURE 2 | Classification of miRNAs altered by ischemia. The figure shows the distribution of the miRNAs altered by ischemia relative to control in male and female mouse cortex. For initial studies we focused on miRNAs that were significantly decreased $(<-1.5 S D)$ or increased $(>1.5 S D)$ in the male and female mouse cortex.

a significant difference between miR-15b* and miR-125b-3p ( $p<$ 0.0001 ), and (3) there was no interaction between the experimental condition and the miRNAs $(p=0.1272)$. The within subject matching is significant $(p=0.0004)$, suggesting that variation across is due to individual variation, not to chance. These analyses support that neither miR-125b-1-3p nor miR-15b* is sensitive to the experimental manipulations (Figure 3) in males (3A) or females (3B), thus the average of both was used as a normalizing factor.

We then examined whether any of the selected miRNAs were sensitive to the sham surgery. Since the original miRNA array comparison was performed on control vs. ischemic conditions, we examined whether there was any significant influence of a surgical sham on miRNA expression. Thus, we compared the normalized $\mathrm{Ct}$ values $(\Delta \mathrm{Ct})$ for each miRNA in Control and Sham experimental groups using a 2-Way repeated measures ANOVA (Prism, GraphPad Software). The results show: (1) there is no effect of the experimental group $(p=0.1130),(2)$ there is a significant effect of miRNA $(p<0.0001)$, (3) there is no interaction between miRNA and experimental group $(p=0.1344)$, and (4) there is a significant within-subject matching $(p=0.0116)$, suggesting variations are due to individual subject variation, rather than random. The conclusions from these analyses are that there is no influence of the surgery alone on any of the miRNAs examined here. Thus, Figure 4 shows the changes in select miRNAs expressed in male (Figure 4A) of female (Figure 4B) cortex in control vs. ischemic treated cortex.

After accounting for the above, the results show that many but not all of the miRNAs under study showed changes in the same direction (up or down) in the individual qRT-PCR assays, consistent with the changes in expression detected by the pooled samples in the miRNA arrays (Figure 5). MiR-686 and miR-1224 both showed increased expression in males and females, as predicted by the miRNA arrays. In addition, miR-296-5p decreased 
Table 1 | MicroRNAs Regulated By Ischemia.

\begin{tabular}{|c|c|c|c|c|c|c|c|c|}
\hline Male & \multicolumn{2}{|c|}{$\Delta \Delta \mathbf{C t}$} & Male and Female & \multicolumn{2}{|c|}{$\Delta \Delta \mathbf{C t}$} & Female & \multicolumn{2}{|c|}{$\Delta \Delta \mathbf{C t}$} \\
\hline $\operatorname{miR}-19 a^{*}$ & -2.57 & -0.23 & miR-296-5p & -2.16 & -2.76 & $m i R-509-3 p$ & 2.03 & -2.35 \\
\hline $\operatorname{miR}-145^{*}$ & -2.68 & -1.41 & & & & miR-543 & -1.70 & -1.79 \\
\hline miR-450b-3p & -2.32 & 0.22 & & & & miR-741 & -0.22 & -1.87 \\
\hline miR-207 & 2.22 & 1.64 & miR-27a* & 4.06 & 3.94 & miR-197 & 1.30 & 2.47 \\
\hline $\operatorname{miR}-218-2^{*}$ & 2.37 & 1.06 & miR-135a* & 3.95 & 2.56 & miR-200c* & 1.51 & 1.95 \\
\hline miR-327 & 3.17 & 1.57 & miR-196a* & 2.79 & 1.93 & miR-466f-3p & 1.58 & 2.03 \\
\hline miR-345-3p & 2.26 & 0.76 & miR-200a* & 2.59 & 2.13 & miR-466f-5p & 1.06 & 2.08 \\
\hline miR-466g & 2.33 & 1.29 & miR-200b* & 2.72 & 2.10 & miR-615-3p & 0.18 & 2.47 \\
\hline $\operatorname{miR}-675-3 p$ & 2.90 & 0.73 & miR-466i & 1.98 & 1.79 & miR-883b-5p & 0.93 & 2.88 \\
\hline miR-682 & 2.12 & -0.41 & $\operatorname{miR}-470 *$ & 3.30 & 2.37 & & & \\
\hline miR-697 & 2.27 & -0.09 & miR-483* & 4.84 & 3.70 & & & \\
\hline miR-770-5p & 2.10 & 1.33 & miR-546 & 2.03 & 2.32 & & & \\
\hline miR-1187 & 2.16 & 0.20 & miR-681 & 1.95 & 2.16 & & & \\
\hline miR-1190 & 2.66 & 1.12 & miR-615-5p & 3.40 & 2.22 & & & \\
\hline miR-1892 & 3.06 & 1.22 & miR-654-3p & 4.03 & 3.88 & & & \\
\hline miR-1896 & 2.18 & 0.70 & miR-677 & 2.78 & 4.10 & & & \\
\hline \multirow[t]{8}{*}{ miR-1897-5p } & 1.97 & 0.02 & miR-678 & 2.87 & 2.60 & & & \\
\hline & & & miR-684 & 2.03 & 3.86 & & & \\
\hline & & & miR-685 & 5.74 & 4.60 & & & \\
\hline & & & miR-1196 & 4.98 & 4.56 & & & \\
\hline & & & miR-1199 & 5.41 & 4.95 & & & \\
\hline & & & miR-1224 & 4.17 & 3.67 & & & \\
\hline & & & miR-1895 & 3.58 & 2.46 & & & \\
\hline & & & miR-1897-3p & 6.67 & 5.09 & & & \\
\hline
\end{tabular}

MiRNAs significantly regulated by ischemia in male and female mouse brain.

(A) Lists the $\Delta \Delta$ Cts for miRNAs significantly decreased in ischemia $(<-1.5 S D)$ in male and female cortex.

(B) Lists the $\Delta \Delta$ Cts for miRNAs significantly increased by ischemia (>1.5 SD) in male and female cortex.

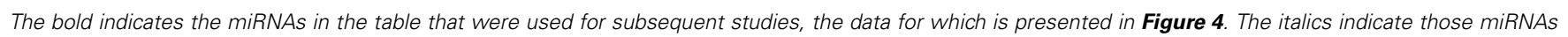
that were found to be oppositely regulated in male and female brain.

in males and females, and miR-125b-3p showed no changes in male or female brain, consistent with the miRNA arrays. MiR682 showed increased expression in males, consistent with the miRNA arrays, but there was increased expression in females that was opposite from the arrays. MiR-883a-3p showed increased expression in females, consistent with the miRNA arrays, but there was a slight increase in the expression in males relative to the small decrease in expression that was detected in the arrays.
Also, miR-509-3p increased in the males, consistent with the miRNA arrays, but decreased significantly in the females which was opposite from the arrays.

These outcomes support that miRNAs are regulated by ischemia in male and female brain. However, they also show that the use of different array formats, or pooled vs. individual qRT-PCR samples, likely influences the expression results (Git et al., 2010). The miRNAs with the most robust changes in 


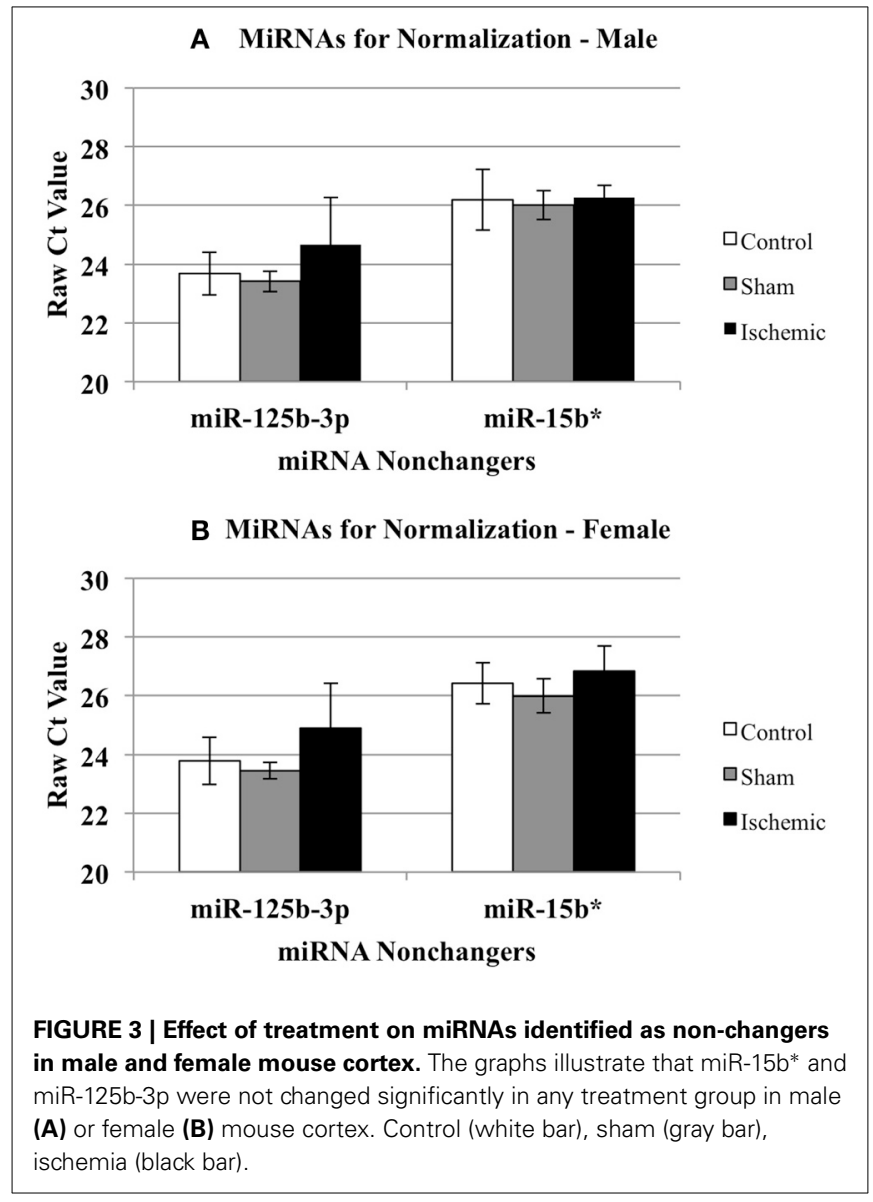

expression in the arrays showed consistent changes in the individual arrays, particularly for those miRNAs that increased in expression. This observation suggests that decreases in miRNA expression from RNA degradation, low abundance of miRNAs, or pooling samples, may affect the array outcomes and data interpretation.

\section{MICRORNA TARGET PREDICTION}

We used the miRmap target prediction program (http://mirmap. ezlab.org/) to query the predicted targets of miRNAs increased by ischemia in male and female brain (Vejnar et al., 2013). We used miRmap to query the targets of the 17 miRNAs increased by ischemia in male brain. The results show target genes and the top number of miRNAs predicted to target this gene with a cutoff of 6 (Table 2A). We also used miRmap to query the targets of the 9 miRNAs increased by ischemia in female brain. The results show target genes and the top number of miRNAs predicted to target this gene with a cutoff of 4 (Table 2B). The results show that the miRNAs increased by ischemia in male and female brain are distinct, and only 2 targets are detected in common in the groups, CD73 and PKN2. These findings suggest that differential pathways are targeted by miRNAs increased by ischemia and that these pathways may lead to differential outcomes to ischemia may underlie the sexually dimorphic responses to ischemia, wherein females are afforded greater protection against ischemia insult

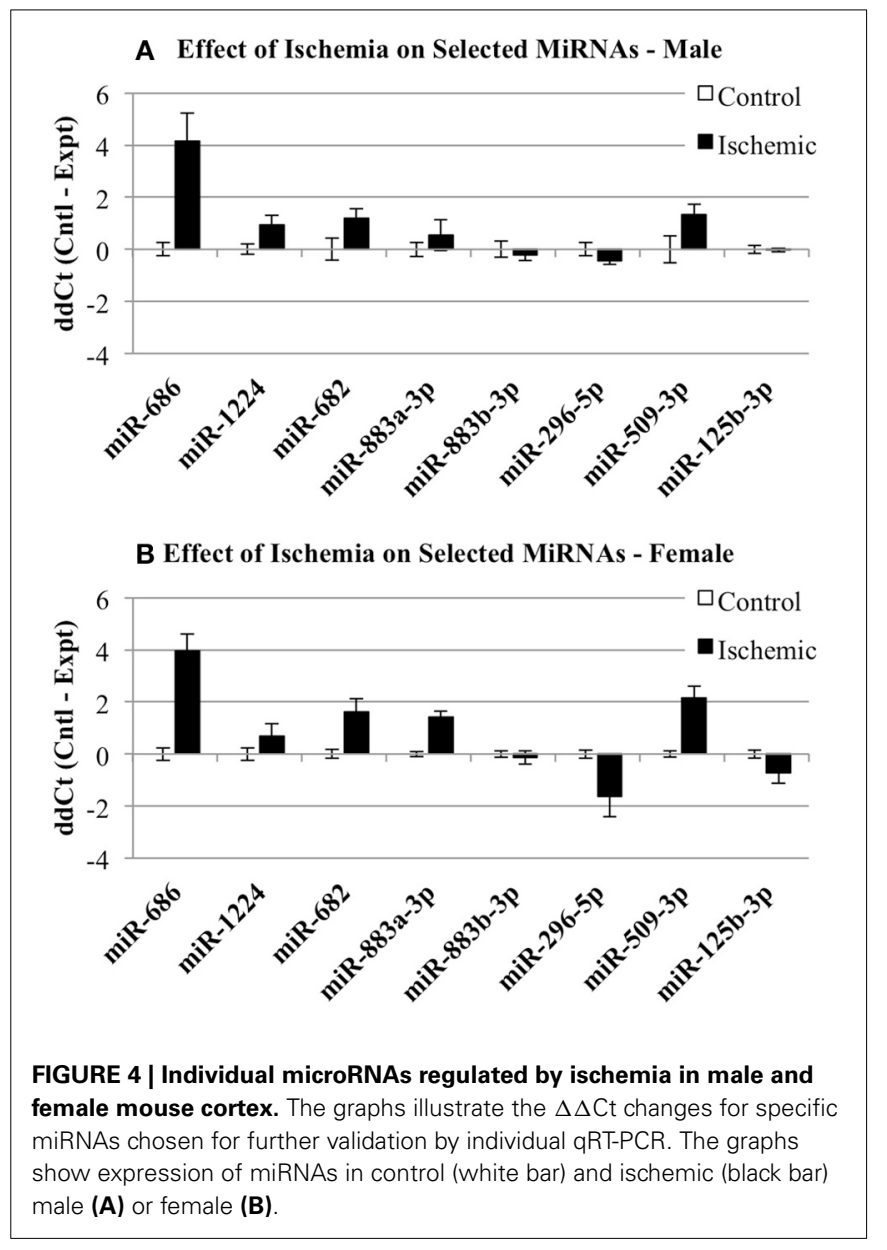

than males. PANTHER target analysis revealed that there are 36 pathways of the genes targeted by miRNAs increased in male brain, and there are 69 pathways for the genes targeted by miRNAs in the female brain (Table 3-Supplemental Data). These findings support that distinct pathways are targeted by miRNA responses to ischemia, and provide an opportunity to focus studies on specific protein and protein pathways that may have been overlooked in prior studies.

\section{DISCUSSION}

Previous studies have shown that miRNAs are regulated in the brain in response to stress, including cerebral ischemia (Fasanaro et al., 2010; Saugstad, 2010; Rink and Khanna, 2011; Liu et al., 2013; Ouyang et al., 2013). However, to our knowledge, we are the first to show miRNA responses to ischemia that are sex dependent, i.e., there are differential responses in male or female brain following ischemia. For this study we focused on those miRNAs differentially expressed between males and females greater than $1.5 S D$ from the mean $\Delta \Delta \mathrm{Ct}$ in order to look at the most robust changers in response to ischemia. However, mRNAs can be targeted by many miRNAs (Doench and Sharp, 2004) and even small, subtle changes in miRNA levels can lead to significant changes in mRNA translation or stability. Thus, we are currently evaluating those differentially expressed miRNAs that 


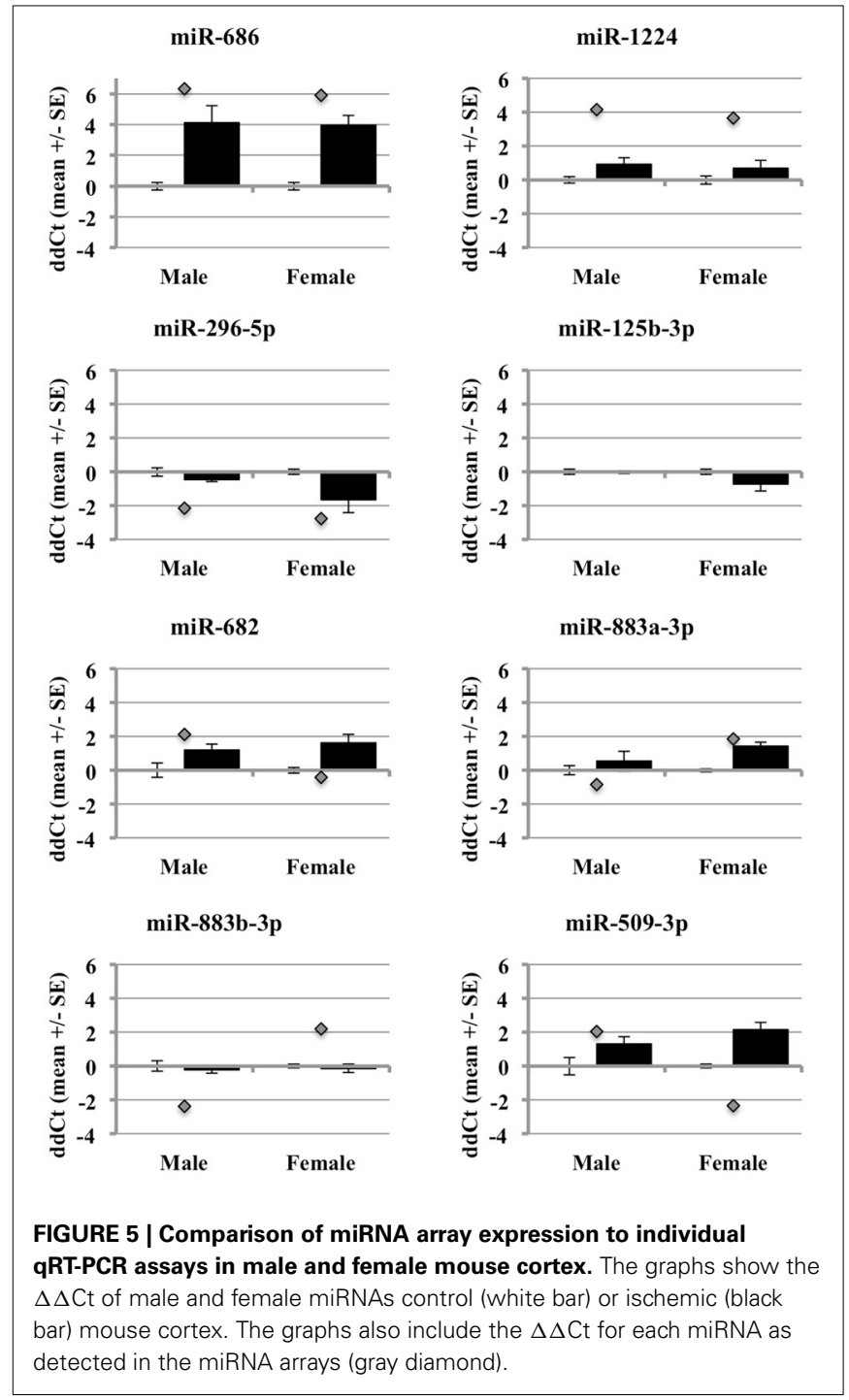

show a change between 1.0 and $1.5 S D$ from the mean to identify additional proteins/pathways that could underlie differential responses to ischemia in male and female brain.

Our studies also revealed a signature miRNA response to ischemia that is common to both males and females. These key findings provide a mechanism, based on differential miRNA expression in male and female brain, to identify cellular or molecular targets that could underlie the sex differences in responses to ischemia. Such insight is likely to have implications for therapeutic strategies for the treatment of stroke in men vs. women. In addition, these studies also provide the ability to examine new targets that might contribute to ischemic injury in both males and females. Thus, we are focused on identifying the cellular/molecular targets of the ischemic-regulated miRNAs to determine how these miRNAs produce differential outcomes to ischemic injury. The genes identified in this study as predicted target of the miRNAs increased by ischemia in male and female ischemic cortex (Table 2) are quite distinct, and support that these miRNAs are likely initiating changes in distinct proteins and pathways that lead to altered phenotypes in in response to
Table 2 | Predicted Proteins Targeted By MicroRNAs Significantly Increased Following Ischemia.

\begin{tabular}{|c|c|c|c|c|c|}
\hline \multirow{2}{*}{\multicolumn{2}{|c|}{$\begin{array}{c}\text { (A) Male } \\
\text { Of } 17 \text { Increased } \\
\text { microRNAs }\end{array}$}} & \multirow{2}{*}{\multicolumn{4}{|c|}{$\begin{array}{c}\text { (B) Female } \\
\text { Of } 9 \text { Increased } \\
\text { microRNAs }\end{array}$}} \\
\hline & & & & & \\
\hline Gene & $\begin{array}{c}\text { No. of } \\
\text { microRNAs }\end{array}$ & Gene & $\begin{array}{c}\text { No. of } \\
\text { microRNAs }\end{array}$ & Gene & $\begin{array}{c}\text { No. of } \\
\text { microRNAs }\end{array}$ \\
\hline Brwd3 & 9 & Cdc73 & 6 & Lyvel & 4 \\
\hline Ccr3 & 7 & $\mathrm{Cnrl}$ & 6 & Mbtps2 & 4 \\
\hline Cdc73 & 7 & Cadm2 & 5 & Mklnl & 4 \\
\hline Clintl & 7 & Cbln2 & 5 & Mon2 & 4 \\
\hline Em15 & 7 & Lcorl & 5 & Naa50 & 4 \\
\hline Fam55c & 7 & Pkn2 & 5 & Nucksl & 4 \\
\hline Map3k2 & 7 & S1c30a4 & 5 & Odzl & 4 \\
\hline Mef2c & 7 & S1c6a14 & 5 & Ogfrll & 4 \\
\hline Ano3 & 6 & Slitrk4 & 5 & Prrg3 & 4 \\
\hline Atp $13 a 3$ & 6 & Strbp & 5 & Pura & 4 \\
\hline Atp2c1 & 6 & Taf4 a & 5 & Ragl & 4 \\
\hline Ccdc93 & 6 & Akap2 & 4 & Rbpms2 & 4 \\
\hline Cnot61 & 6 & Ap4e 1 & 4 & Rhoa & 4 \\
\hline Coll lal & 6 & Arl5b & 4 & Rqcdl & 4 \\
\hline Csmdl & 6 & Atadl & 4 & Sec24a & 4 \\
\hline Dio2 & 6 & Atpafl & 4 & Serpinb7 & 4 \\
\hline Edaradd & 6 & Cblb & 4 & Slitrkl & 4 \\
\hline Eif2c3 & 6 & Cd200r4 & 4 & Sox4 & 4 \\
\hline Fgfbp3 & 6 & Clec2h & 4 & Sox5 & 4 \\
\hline Gabrg3 & 6 & Cltc & 4 & Syngr3 & 4 \\
\hline Ghr & 6 & Crebzf & 4 & Tbc1d24 & 4 \\
\hline Gng2 & 6 & Cxxc4 & 4 & Tdrd5 & 4 \\
\hline Hipk3 & 6 & Ect2 & 4 & Tecrl & 4 \\
\hline Hmgcsl & 6 & Femlb & 4 & Tmem236 & 4 \\
\hline $\mathrm{Htrl} \mathrm{a}$ & 6 & Flil & 4 & Trip 12 & 4 \\
\hline IIrapll & 6 & Foxal & 4 & Ubrl & 4 \\
\hline 1121 & 6 & Gas7 & 4 & Ugp2 & 4 \\
\hline Mbn12 & 6 & Gnal3 & 4 & Usp38 & 4 \\
\hline Ms4a4c & 6 & Gngtl & 4 & Usp46 & 4 \\
\hline Mtmr6 & 6 & Golga7 & 4 & Usp9x & 4 \\
\hline Nlgn3 & 6 & Gopc & 4 & Vmn1r7 & 4 \\
\hline Oxtr & 6 & Gpr149 & 4 & Xpo7 & 4 \\
\hline Pkn2 & 6 & Grik2 & 4 & Ywhag & 4 \\
\hline Ppmll & 6 & Hipl & 4 & Zdbf2 & 4 \\
\hline Rora & 6 & Ikzf2 & 4 & Zfand6 & 4 \\
\hline Sdpr & 6 & & & & \\
\hline
\end{tabular}

Proteins predicted to be targeted by miRNAs differentially regulated by ischemia in male and female brain. The table lists show the gene name and the top number of miRNAs in each group predicted to target the gene (6-9 of 17 miRNAs in male, 4-6 miRNAs in female).

ischemia in male and female brain. PANTHER pathway analysis of the gene targets of miRNAs increased by ischemia in male and female (Table 3-Supplemental Data) support that distinct pathways are induced in each gender, and future studies are focused on clarifying the importance of these pathways in differential responses to ischemia in male and female brain. 
One limitation of the present studies is that miRNA target prediction is still evolving. However, we used the miRmap target prediction program (http://mirmap.ezlab.org/) to query the predicted targets of miRNAs increased by ischemia in male and female brain (Vejnar et al., 2013). The miRmap open source software library employs eleven predictor features, three of which are novel, as well as common features of target prediction including thermodynamic, evolutionary, probabilistic, and sequence-based features. This program allows the examination of feature correlations and comparison of their predictive power in an unbiased way using high throughput experimental data. Overall, target site accessibility appears to be the most predictive feature. Methods for identifying real miRNA targets are also evolving, such as using RISCtraps to stabilize and purify targets of specific miRNAs (Cambronne et al., 2012), but will be essential for future studies identifying the targets of the miRNAs regulated by ischemia in male and female brain. Another limitation is that we examined miRNA expression in the whole ischemic cortex, which may have diluted the expression of distinct miRNAs due to inclusion of both the core and penumbra. However, at $8 \mathrm{~h}$ reperfusion time, there are no apparent changes in cell death and thus no reliable method to indicate the boundary between core and penumbra. Thus, we will examine the regional/cellular changes in differentially expressed miRNAs to identify their potential role as mediators of cell death in the ischemic core.

In conclusion, we have shown that the sex-related differences in the response to ischemic insult observed in males and female mice is also characterized by a corresponding difference in expression levels of a specific set of miRNAs. Thus, current and future studies in our laboratory are focused on elucidating the roles of ischemia-regulated miRNAs that show distinct changes in male or female brain, along with those miRNAs that are regulated in a sex-independent fashion. We anticipate that these studies will clarify the mechanisms underlying responses to ischemia, both sex-related and non-sex-related. We also trust that they will provide guidance for the future design of therapeutic strategies to treat stroke specifically tailored to male and female patients. For example, a recent study of miRNAs in cardiac ischemia revealed that cardiomyocyte proliferation could be stimulated by the exogenous administration of miRNAs, and more importantly, that this treatment could restore cardiac mass and promote functional recovery after myocardial infarction in adult rats (Eulalio et al., 2012). We propose that future therapies could similarly be developed for cerebral ischemia, whereby administration of miRNAs designed specifically for males or females would promote functional recovery after stroke in humans.

\section{AUTHOR CONTRIBUTIONS}

Stephanie J. Murphy and Julie A. Saugstad conceptualized the project. Yingxin Chen performed all of the mouse middle cerebral artery occlusion surgeries and brain sub-dissections. Simon J. Thompson, Catherine M. Davis, and Jennifer M. Young isolated the RNAs and performed the SABiosciences miRNA arrays. Theresa A. Lusardi and Julie A. Saugstad analyzed the miRNA array data. Jay I. Phillips isolated RNA and performed the individual qRT-PCR assays. Theresa A. Lusardi, Julie A. Saugstad, and Jay I. Phillips analyzed the miRNA qRT-PCR data. Theresa
A. Lusardi, Stephanie J. Murphy, and Julie A. Saugstad prepared the manuscript.

\section{ACKNOWLEDGMENTS}

This work was support by the National Institutes of Health (R21NS078581, Stephanie J. Murphy; R01NS064270, Julie A. Saugstad).

\section{SUPPLEMENTARY MATERIAL}

The Supplementary Material for this article can be found online at: http://www.frontiersin.org/journal/10.3389/fnmol.2014. 00011/abstract

\section{REFERENCES}

Ambros, V. (2004). The functions of animal micrornas. Nature 431, 350-355. doi: 10.1038/nature02871

Appelros, P., Stegmayr, B., and Terent, A. (2009). Sex differences in stroke epidemiology: a systematic review. Stroke 40, 1082-1090. doi: 10.1161/STROKEAHA.108.540781

Banerjee, A., Wang, J., Bodhankar, S., Vandenbark, A. A., Murphy, S. J., and Offner, H. (2013). Phenotypic changes in immune cell subsets reflect increased infarct volume in male vs. female mice. Trans. Stroke Res. 4, 554-563. doi: 10.1007/s12975-013-0268-Z

Bushnell, C. D. (2008). Stroke and the female brain. Nat. Clin. Pract. Neurol. 4, 22-33. doi: 10.1038/ncpneuro0686

Cambronne, X. A., Shen, R., Auer, P. L., and Goodman, R. H. (2012). Capturing microrna targets using an rna-induced silencing complex (risc)-trap approach. Proc. Natl. Acad. Sci. U.S.A. 109, 20473-20478. doi: 10.1073/pnas.1218887109

Chen, Y., Bodhankar, S., Murphy, S. J., Vandenbark, A. A., Alkayed, N. J., Offner, H. (2012). Intrastriatal B-cell administration limits infarct size after stroke in B-cell deficient mice. Metab. Brain Dis. 27, 487-493. doi: 10.1007/s11011-012-9317-7

Cheng, J., and Hurn, P. D. (2010). Sex shapes experimental ischemic brain injury. Steroids 75, 754-759. doi: 10.1016/j.steroids.2009.10.014

Chua, J. H., Armugam, A., and Jeyaseelan, K. (2009). Micrornas: biogenesis, function and applications. Curr. Opin. Mol. Ther. 11, 189-199.

Dharap, A., Bowen, K., Place, R., Li, L. C., and Vemuganti, R. (2009). Transient focal ischemia induces extensive temporal changes in rat cerebral micrornaome. J. Cereb. Blood Flow Metab. 29, 675-687. doi: 10.1038/jcbfm.2008.157

Doench, J. G., and Sharp, P. A. (2004). Specificity of microrna target selection in translational repression. Genes Dev. 18, 504-511. doi: 10.1101/gad.1184404

Eulalio, A., Mano, M., Dal Ferro, M., Zentilin, L., Sinagra, G., Zacchigna, S., et al. (2012). Functional screening identifies miRNAs inducing cardiac regeneration. Nature 492, 376-381. doi: 10.1038/nature11739

Fasanaro, P., Greco, S., Ivan, M., Capogrossi, M. C., and Martelli, F. (2010). Microrna: emerging therapeutic targets in acute ischemic diseases. Pharmacol. Ther. 125, 92-104. doi: 10.1016/j.pharmthera.2009.10.003

Feng, W., and Feng, Y. (2011). Micrornas in neural cell development and brain diseases. Sci. China Life Sci. 54, 1103-1112. doi: 10.1007/s11427-011-4249-8

Git, A., Dvinge, H., Salmon-Divon, M., Osborne, M., Kutter, C., Hadfield, J., et al. (2010). Systematic comparison of microarray profiling, real-time pcr, and next-generation sequencing technologies for measuring differential microrna expression. RNA 16, 991-1006. doi: 10.1261/rna.1947110

Guarnieri, D. J., and DiLeone, R. J. (2008). Micrornas: a new class of gene regulators. Ann. Med. 40, 197-208. doi: 10.1080/07853890701771823

Ilnytskyy, Y., Zemp, F. J., Koturbash, I., and Kovalchuk, O. (2008). Altered microrna expression patterns in irradiated hematopoietic tissues suggest a sexspecific protective mechanism. Biochem. Biophys. Res. Commun. 377, 41-45. doi: 10.1016/j.bbrc.2008.09.080

Jeyaseelan, K., Lim, K. Y., and Armugam, A. (2008). Microrna expression in the blood and brain of rats subjected to transient focal ischemia by middle cerebral artery occlusion. Stroke 39, 959-966. doi: 10.1161/strokeaha.107.500736

Johnsen, D., and Murphy, S. J. (2011). Isoflurane preconditioning protects neurons from male and female mice against oxygen and glucose deprivation and is modulated by estradiol only in neurons from female mice. Neuroscience 199, 368-374. doi: 10.1016/j.neuroscience.2011.09.053 
Kitano, H., Young, J. M., Cheng, J., Wang, L., Hurn, P. D., and Murphy, S. J. (2007). Gender-specific response to isoflurane preconditioning in focal cerebral ischemia. J. Cereb. Blood Flow Metab. 27, 1377-1386. doi: 10.1038/sj.jcbfm.9600444

Koerner, I., Murphy, S. J., and Hurn, P. D. (2007). "Gender, sex steroids, and cerebral ischemic pathobiology," in Acute Ischemic Injury and Repair in the Nervous System, ed P. Chan (New York, NY: Kluwer Academic/Plenum Publishers), 186-207.

Koturbash, I., Zemp, F., Kolb, B., and Kovalchuk, O. (2011). Sex-specific radiation-induced micrornaome responses in the hippocampus, cerebellum and frontal cortex in a mouse model. Mutat. Res. 722, 114-118. doi: 10.1016/j.mrgentox.2010.05.007

Lang, J. T., and McCullough, L. D. (2008). Pathways to ischemic neuronal cell death: are sex differences relevant? J. Transl. Med. 6, 33. doi: 10.1186/1479-5876-6-33

Li, H., Pin, S., Zeng, Z., Wang, M. M., Andreasson, K. A., and McCullough, L. D. (2005). Sex differences in cell death. Ann. Neurol. 58, 317-321. doi: 10.1002/ana.20538

Liu, D. Z., Tian, Y., Ander, B. P., Xu, H., Stamova, B. S., Zhan, X., et al. (2010). Brain and blood microrna expression profiling of ischemic stroke, intracerebral hemorrhage, and kainate seizures. J. Cereb. Blood Flow Metab. 30, 92-101. doi: 10.1038/jcbfm.2009.186

Liu, M., Hurn, P. D., Roselli, C. E., and Alkayed, N. J. (2007). Role of p450 aromatase in sex-specific astrocytic cell death. J. Cereb. Blood Flow Metab. 27, 135-141. doi: 10.1038/sj.jcbfm.9600331

Liu, M., Oyarzabal, E. A., Yang, R., Murphy, S. J., and Hurn, P. D. (2008). A novel method for assessing sex-specific and genotype-specific response to injury in astrocyte culture. J. Neurosci. Methods 171, 214-217. doi: 10.1016/j.jneumeth.2008.03.002

Liu, X. S., Chopp, M., Zhang, R. L., and Zhang, Z. G. (2013). Micrornas in cerebral ischemia-induced neurogenesis. J. Neuropathol. Exp. Neurol. 72, 718-722. doi: 10.1097/NEN.0b013e31829e4963

Lusardi, T. A., Farr, C. D., Faulkner, C. L., Pignataro, G., Yang, T., Lan, J., et al. (2010). Ischemic preconditioning regulates expression of micrornas and a predicted target, mecp2, in mouse cortex. J. Cereb. Blood Flow Metab. 30, 744-756. doi: $10.1038 /$ jcbfm.2009.253

Lusardi, T. A., Thompson, S. J., Macdonald, I. C., Lan, J. Q., Theofilas, P., and Saugstad, J. A. (2012). Effect of (s)-3,5-dhpg on microrna expression in mouse brain. Exp. Neurol. 235, 497-507. doi: 10.1016/j.expneurol.2012.01.018

Mi, H., Muruganujan, A., Casagrande, J. T., and Thomas, P. D. (2013). Large-scale gene function analysis with the panther classification system. Nat. Protoc. 8, 1551-1566. doi: 10.1038/nprot.2013.092

Mi, H., and Thomas, P. (2009). Panther pathway: an ontology-based pathway database coupled with data analysis tools. Methods Mol. Biol. 563, 123-140. doi: 10.1007/978-1-60761-175-2_7

Murchison, E. P., and Hannon, G. J. (2004). MiRNAs on the move: miRNA biogenesis and the rnai machinery. Curr. Opin. Cell Biol. 16, 223-229. doi: 10.1016/j.ceb.2004.04.003

Murphy, S. J., McCullough, L. D., and Smith, J. M. (2004). Stroke in the female: role of biological sex and estrogen. ILAR J. 45, 147-159. doi: 10.1093/ilar.45.2.147

Niwa, R., and Slack, F. J. (2007). The evolution of animal microrna function. Curr. Opin. Genet. Dev. 17, 145-150. doi: 10.1016/j.gde.2007.02.004

Ouyang, Y. B., Stary, C. M., Yang, G. Y., and Giffard, R. (2013). Micrornas: innovative targets for cerebral ischemia and stroke. Curr. Drug Targets 14, 90-101. doi: $10.2174 / 138945013804806424$
Ovbiagele, B., Goldstein, L. B., Higashida, R. T., Howard, V. J., Johnston, S. C., Khavjou, O. A., et al. (2013). Forecasting the future of stroke in the united states: a policy statement from the american heart association and american stroke association. Stroke 44, 2361-2375. doi: 10.1161/STR.0b013e31829 $734 \mathrm{f} 2$

Persky, R. W., Turtzo, L. C., and McCullough, L. D. (2010). Stroke in women: disparities and outcomes. Curr. Cardiol. Rep. 12, 6-13. doi: 10.1007/s11886-0090080-2

Reeves, M. J., Bushnell, C. D., Howard, G., Gargano, J. W., Duncan, P. W., Lynch, G., et al. (2008). Sex differences in stroke: epidemiology, clinical presentation, medical care, and outcomes. Lancet Neurol. 7, 915-926. doi: 10.1016/S14744422(08)70193-5

Rink, C., and Khanna, S. (2011). MicroRNA in ischemic stroke etiology and pathology. Physiol. Genomics. 43, 521-528. doi: 10.1152/physiolgenomics.00158.2010

Saini, M., and Shuaib, A. (2008). Stroke in women. Recent Pat. Cardiovasc. Drug Discov. 3, 209-221. doi: 10.2174/157489008786264032

Saugstad, J. A. (2010). Micrornas as effectors of brain function with roles in ischemia and injury, neuroprotection, and neurodegeneration. J. Cereb. Blood Flow Metab. 30, 1564-1576. doi: 10.1038/jcbfm.2010.101

Siegel, C., Li, J., Liu, F., Benashski, S. E., and McCullough, L. D. (2011). Mir-23a regulation of $\mathrm{x}$-linked inhibitor of apoptosis (xiap) contributes to sex differences in the response to cerebral ischemia. Proc. Natl. Acad. Sci. U.S.A. 108, 11662-11667. doi: 10.1073/pnas.1102635108

Siegel, C., Turtzo, C., and McCullough, L. D. (2010). Sex differences in cerebral ischemia: possible molecular mechanisms. J. Neurosci. Res. 88, 2765-2774. doi: 10.1002/jnr.22406

Tan, J. R., Koo, Y. X., Kaur, P., Liu, F., Armugam, A., Wong, P. T., et al. (2011) Micrornas in stroke pathogenesis. Curr. Mol. Med. 11, 76-92.

Towfighi, A., Markovic, D., and Ovbiagele, B. (2013). Sex differences in revascularization interventions after acute ischemic stroke. J. Stroke Cerebrovasc. Dis. 22, e347-e353. doi: 10.1016/j.jstrokecerebrovasdis.2013.03.018

Vagnerova, K., Koerner, I. P., and Hurn, P. D. (2008). Gender and the injured brain. Anesth. Analg. 107, 201-214. doi: 10.1213/ane.0b013e31817326a5

Vejnar, C. E., Blum, M., and Zdobnov, E. M. (2013). Mirmap web: comprehensive microrna target prediction online. Nucleic Acids Res. 41, W165-W168. doi: $10.1093 /$ nar/gkt430

Conflict of Interest Statement: The authors declare that the research was conducted in the absence of any commercial or financial relationships that could be construed as a potential conflict of interest.

Received: 20 September 2013; accepted: 23 January 2014; published online: 11 February 2014.

Citation: Lusardi TA, Murphy SJ, Phillips JI, Chen Y, Davis CM, Young JM, Thompson SJ and Saugstad JA (2014) MicroRNA responses to focal cerebral ischemia in male and female mouse brain. Front. Mol. Neurosci. 7:11. doi: 10.3389/fnmol.2014.00011 This article was submitted to the journal Frontiers in Molecular Neuroscience.

Copyright (c) 2014 Lusardi, Murphy, Phillips, Chen, Davis, Young, Thompson and Saugstad. This is an open-access article distributed under the terms of the Creative Commons Attribution License (CC BY). The use, distribution or reproduction in other forums is permitted, provided the original author(s) or licensor are credited and that the original publication in this journal is cited, in accordance with accepted academic practice. No use, distribution or reproduction is permitted which does not comply with these terms. 